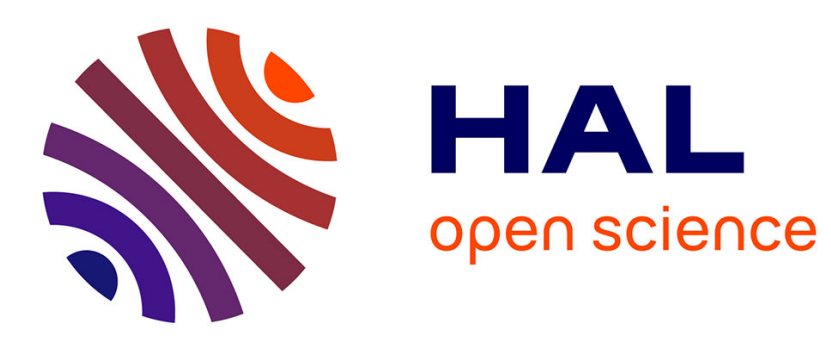

\title{
Les carrières de grès de La Croix-Guillaume à Saint-Quirin (Moselle)
}

Dominique Heckenbenner, Nicolas Meyer

\section{To cite this version:}

Dominique Heckenbenner, Nicolas Meyer. Les carrières de grès de La Croix-Guillaume à Saint-Quirin (Moselle). Gallia - Archéologie de la France antique, 2002, Carrières antiques de la Gaule, 59, pp.145154. 10.3406/galia.2002.3102 . hal-01912512

\section{HAL Id: hal-01912512 https://hal.science/hal-01912512}

Submitted on 20 Jan 2020

HAL is a multi-disciplinary open access archive for the deposit and dissemination of scientific research documents, whether they are published or not. The documents may come from teaching and research institutions in France or abroad, or from public or private research centers.
L'archive ouverte pluridisciplinaire HAL, est destinée au dépôt et à la diffusion de documents scientifiques de niveau recherche, publiés ou non, émanant des établissements d'enseignement et de recherche français ou étrangers, des laboratoires publics ou privés.

\section{(ㅇ)(1) $\$$}

Distributed under a Creative Commons Attribution - NonCommercial - NoDerivatives| 4.0 


\title{
LES CARRIÈRES DE GRÈS
}

DE LA CROIX-GUILLAUME

À SAINT-Quirin (Moselle)

\author{
Dominique Heckenbenner et Nicolas Meyer
}

Mots-clés. Gallo-romain, hameau, carrière, extraction, grès, taille, outils, sculpture.

Key-words. Gallo-Roman, hamlel, quarry, extraction, sandstone, carving, tools, sculpture.

Résumé. Ia fouille du hameau gallo-romain de La Croix-(ruillaume à Saint-Quirin (Moselle) a révélé la présence de plusieurs carrières d'extraction de grès. L'étude exhaustive de la plus vaste d'entre elles a permis d'appréhender les techniques d'extraction utilisées, a stratégie et l'évolution de l'exploitation. Grâce à l'observation des tas de déchets et leur confrontation avec les monuments, les stèles t les statues découverts dans la nécropole et sur l'aire cultuelle, l'hypothèse de l'existence d'un atelier de taille a pu être proposée. Te mobilier découvert montre que les carrières ont été utilisées durant toute la période d'occupation du site. Ces données chronologiques nais aussi l'organisalion topographique du hameau tendent à prouver que ses habitants étaient bien les exploitants des carrières.

Gstract. The excavation of a Gallo-Roman hamlet at La Croix Guillaume (Saint-Quirin, Moselle) revealed several sandstone quarries. I) he extensive study of the largest one enables us to understand quarrying techniques, the strategy of the exploitation and its development.

Through the examination of heaps in correlation with the buildings, the tombstones and the statues of gods found in the cemetery and the sacred area, we can suggest that a workshop was silualed on the sile. The finds show that the quarries zere in activity during the whole lenght of time of occupation. From this dating evidence and the spatial organization of the settlement, it can be assumed that the native people were the quarry contractors.

Situé en forêt domaniale de Saint-Quirin (Moselle), le hameau gallo-romain de La Croix-Guillaume occupe un plateau de $7000 \mathrm{~m}^{2}$ environ, culminant à $487 \mathrm{~m}$, sur les hauteurs boisées des Vosges gréseuses qui dominent à l'est la Sarre rouge et à l'ouest le ruisseau de Saint-Quirin et la Sarre blanche (fig. 112). Le rebord du plateau est relativement abrupt à l'ouest et au nord. En revanche, à l'est et au sud, des terrasses jalonnent les pentes jusqu'à un petit col. Au sud, à une dizaine de kilomètres à vol d'oiseau, se dresse le Donon ; au nord, à la jonction des collines sous-vosgiennes et du plateau lorrain, on rencontre Sarrebourg, l'antique Pons Saravi, dernière étape sur la voie romaine Metz-Strasbourg, avant le col de Saverne.
Géologiquement, cette région correspond à la zone d'affleurement des grès bigarrés du Trias inférieur (Buntsandstein), et plus particulièrement, dans le secteur de La Croix-Guillaume, aux couches intermédiaires. Il s'agit d'un grès micacé de couleur rose, à gros grains, mais comprenant des imprégnations dolomitiques qui le rendent relativement dur (carte géologique Sarrebourg XXXVI-15). Ce substrat rocheux est recouvert sur le plateau d'une couche d'humus peu épaisse $(5$ à $30 \mathrm{~cm}$ environ).

Le hameau de La Croix-Guillaume appartient à un ensemble de sites gallo-romains de hauteur, qui s'étendent au nord de Saverne et de Sarrebourg jusqu'au Donon et dont les nombreux vestiges ont attiré dès le 


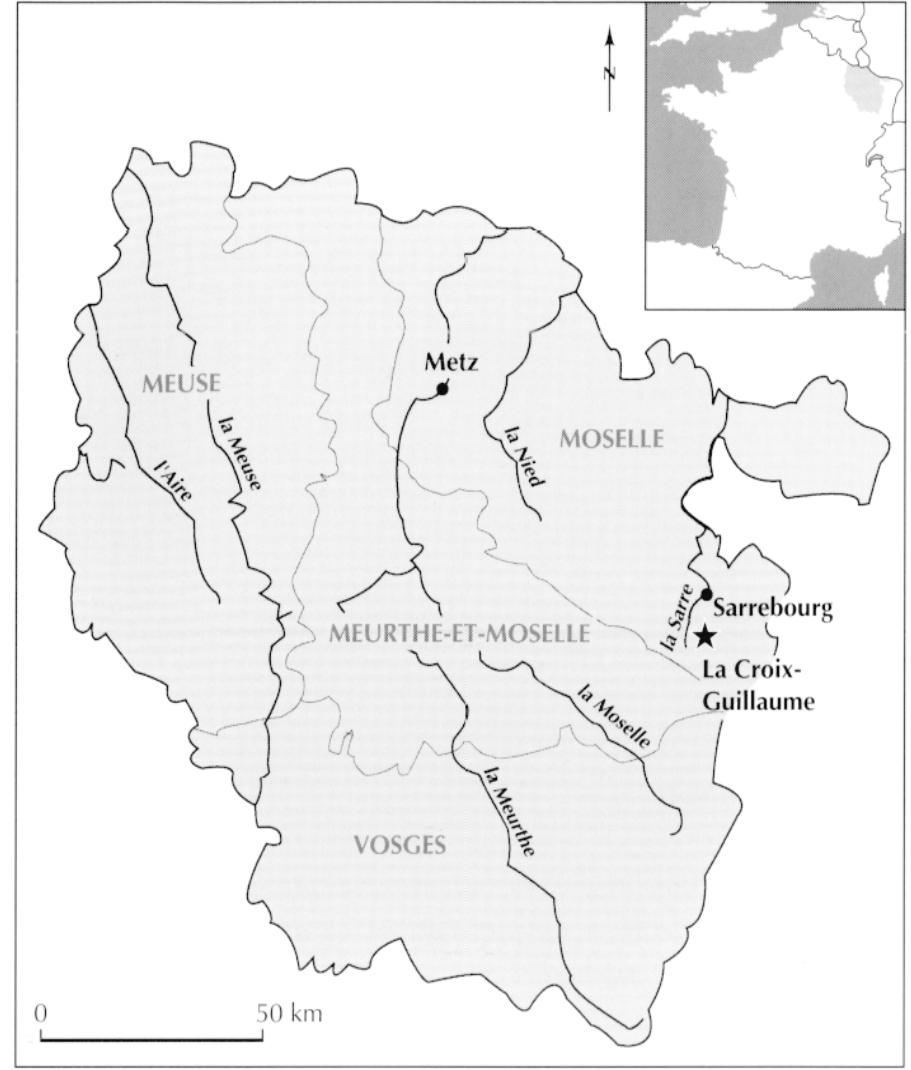

Fig. 112 - Carte de situation de La Croix-(ruillaume à Saint-Quirin, Moselle (DAO D. Heckenbenner, musée de Sarrebourg).

XVIII's. l'attention des érudits. En ce qui concerne plus particulièrement le site de La Croix-Guillaume, il est mentionné brièvement en 1911 (Reusch, 1911, p. 421). En 1962, à la suite de la découverte fortuite de stèles funéraires et votives, une campagne de sondages archéologiques fut conduite par M. Lutz et B. Babault, sous la direction de J.J. Hatt (Gallia, XXII, 2, 1964, p. 356-357 ; Babault, Lutz, 1973). Les observations effectuées sur l'ensemble du site permirent de localiser plusieurs carrières de grès, une habitation et une nécropole aisément repérable grâce aux monuments brisés qui jonchaient le sol.

Une opération programmée fut enfin menée de 1994 à 1999 par Dominique Heckenbenner et Nicolas Meyer. La fouille exhaustive du site et la prospection fine réalisée aux alentours ont révélé la présence de plusieurs carrières d'extraction de grès, d'un petit secteur d'habitat, d'une zone cultuelle et d'une nécropole (fig. 113). Un chemin creux permettait d'accéder au plateau puis se prolongeait par une aire de circulation empierrée. Des bâtiments, situés à l'ouest, ne subsistent que les soubassements en pierre sèche. Les murs et les toitures étaient, de toute évidence, constitués de matériaux légers. Les tessons de céramique découverts dans les fondations indiquent que leur construction a été réalisée postérieurement à 80 après J.-C. Ces maisons s'ouvraient sur une vaste aire empierrée où avaient été édifiées plusieurs stèles de divinités (Jupiter à l'anguipède notamment). La nécropole comprenait 80 structures funéraires (monuments et dépôts d'incinération) regroupées en noyaux. Leurs datations s'échelonnent du milieu du I ${ }^{\text {er }}$ s. au milicu du III ${ }^{\mathrm{e}} \mathrm{s}$. après J.-C. Si l'installation de la nécropole semble précéder la construction des habitations (du moins dans leur état conservé), en revanche, l'ensemble du site a été abandonné dans la seconde moitié du III's. Ces données chronologiques confirment les hypothèses avancées pour les autres sites de hauteur des Vosges (Heckenbenner, Meyer, 1994-1999 et à paraître).

\section{L'EXTRACTION DU GRÈS DANS LES VOSGES PENDANT L'ANTIQUITÉ}

Trois carrières de grès sont mentionnées à proximité de Saverne, une à Reinhardsmunster (Bas-Rhin) au lieudit Moulin de Champagne (Forrer, 1927, p. 314-318; CIL, XIII, 5989), l'autre au Koepfel (Gallia, 36, 2, 1978, p. 370-371), où ont été découvertes des inscriptions émanant de la VIII légion, enfin une troisième au Wasserwald (Gallia, 36, 2, 1978, p. 371). J.J. Hatt signale une carrière à Haslach dans la vallée de la Bruche (Hatt, 1953 , p. 54-55), un atelier de sculpteur près de Hultehouse en Moselle (Gallia, XVIII, 2, 1960, p. 230-231 et XX, 2, 1962, p. 496) et la carrière de La CroixGuillaume à Saint-Quirin (Gallia, XXII, 2, 1964, p. 356-357). Malgré la synthèse réalisée par $\mathbf{R}$. Bedon (1984, p. 44-45), aucune étude plus précise n'a été faite sur ces sites. En revanche, les prospections récentes dans le secteur des Vosges mosellanes ont révélé l'existence de plusieurs exploitations, en général de taille modeste, et notamment aux alentours de Saint-Quirin (Deux-Croix, Sauvageon). Leur origine antique reste cependant à démontrer en l'absence de critères de datation.

L'emploi du grès pour les monuments funéraires et religieux, et pour la construction, est quasi général sur le secteur des Vosges mosellanes, où l'on observe une prédominance du grès des couches intermédiaires. En 


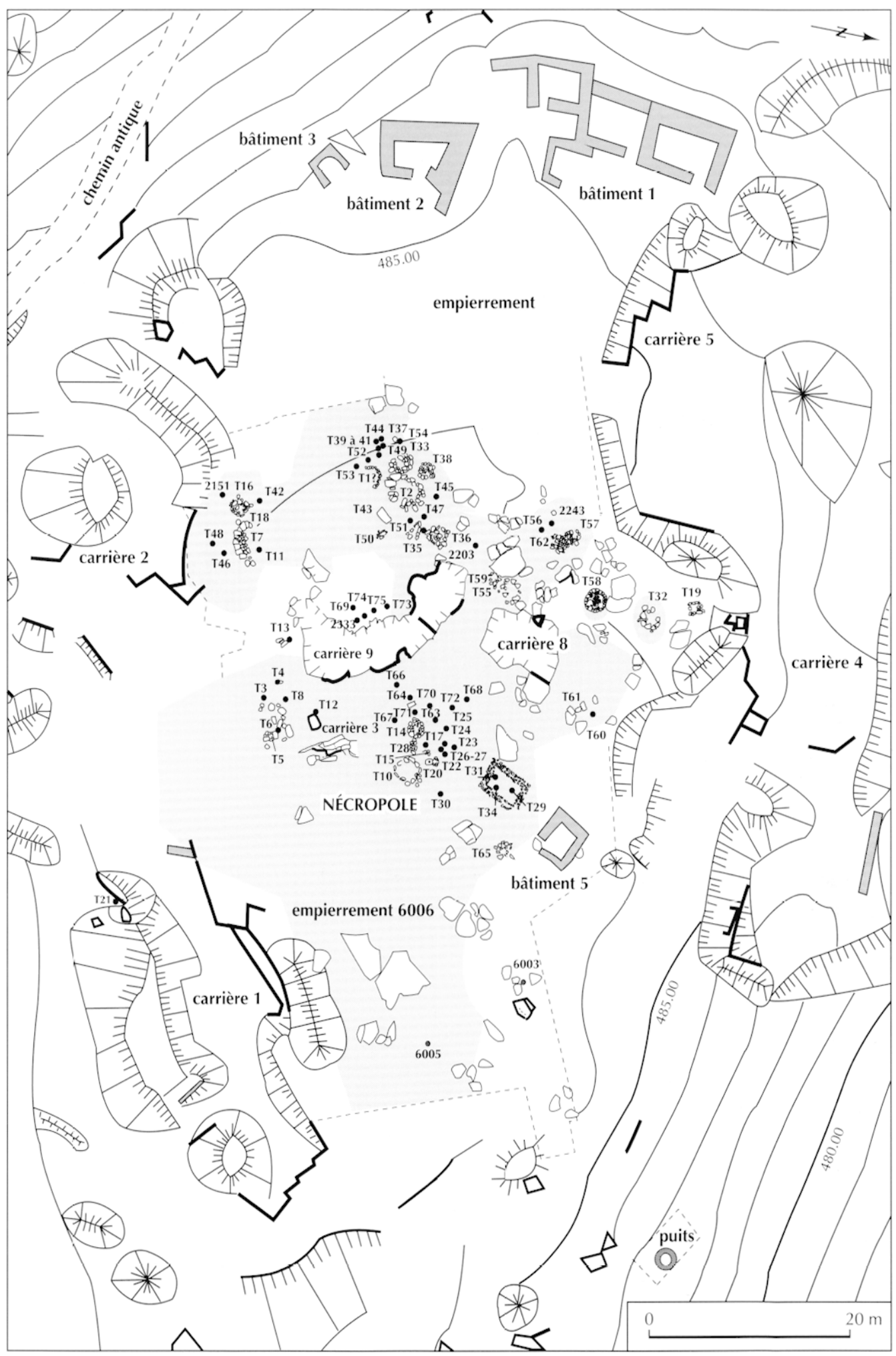

Fig. 113 - Plan du sile de La Croix-Cuillaume ì SaintQuirin, Moselle (DAO N. Meyer, musée de Sarrebourg).

constructions sont généralement réalisées en moellons de calcaire, à l'exclusion des seuils et des éléments d'architecture (colonnes, entablements) taillés dans le grès. 


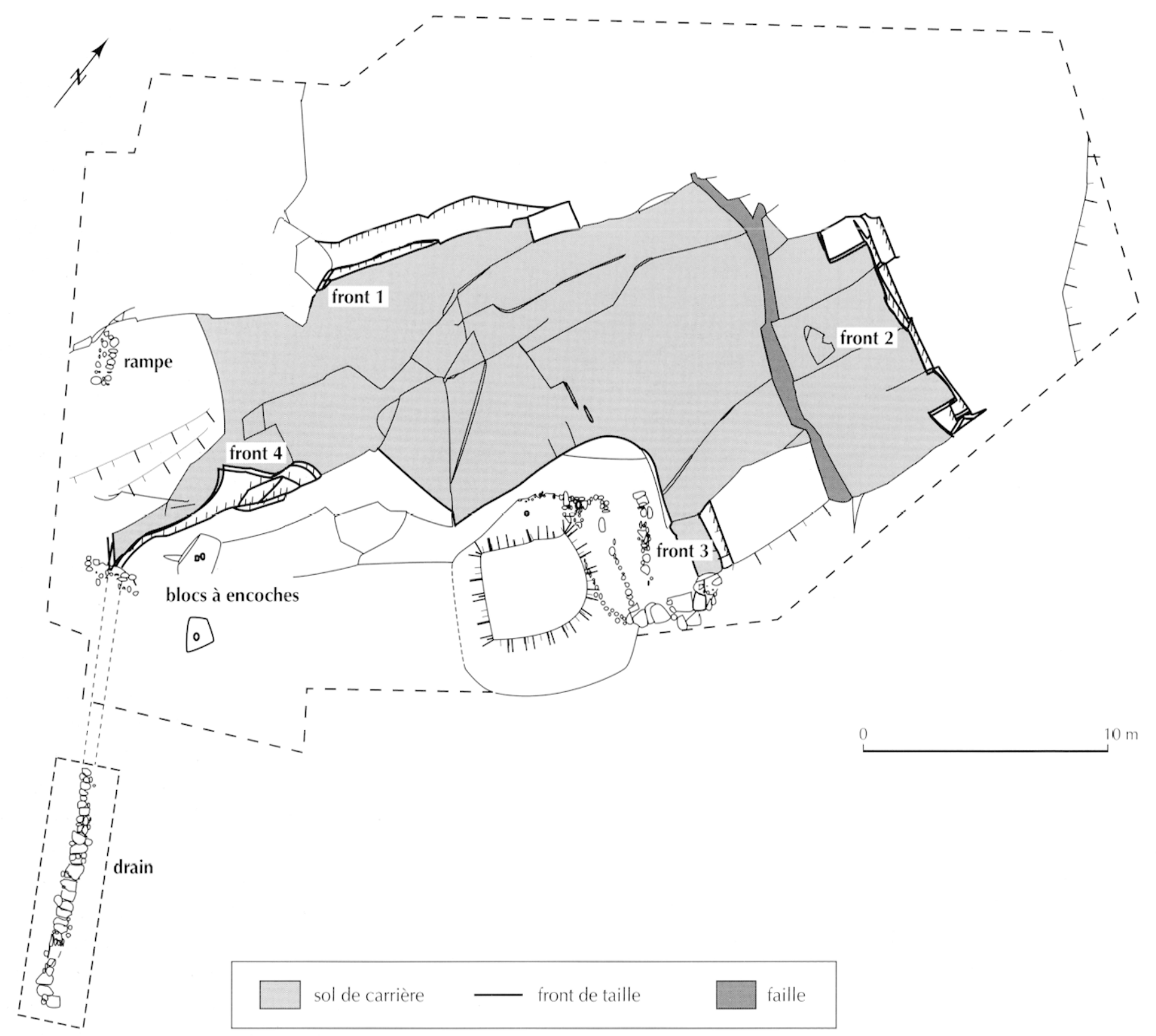

Fig. 114 - Plan de la carrière 1 de La (rroix-Guillaume ì Saint-Quirin, Moselle (I)AO I). Heckenbenner, musée de Sarrebourg).

\section{LES CARRIÈRES DE LA CROIX-GUILLAUME}

Près de cinquante points d'extraction ont été identifiés le long du chemin d'accès ct sur le rebord du plateau. Ces exploitations sont aisément repérables après le dégagement de la végétation, grâce aux traces qu'elles ont laissées: excavations, fronts de taille*, déchets d'extraction. Seules les carrières situées sur le plateau ont fait l'objet d'une étude, en particulier la carric̀re 1 qui a servi de référence. Les autres points d'extraction, de taille modeste et dont l'antiquité n'est pas assurée, ont seulement été cartographiés.

\section{LA CARRIÈRE 1}

Celle-ci s'étend sur environ $1100 \mathrm{~m}^{2}$ au sud du site (fig. 114 et 115). Le chemin qui y mène s'élargit en plateforme en contrebas de la carrière. L'affleurement de grès affecte un léger pendage est-ouest et présente de 
nombreuses failles qui lui donnent par endroit un aspect feuilleté, surtout au nord. Au sud, il se fracture à la rupture de pente. Les tas de déchets sont disposés essentiellement sur les bords sud et nord-est de la carrière. Au centre, le sol de la carrière apparaît sous 10 à $50 \mathrm{~cm}$ d'humus.

\section{DESCRIPTION}

\section{Les fronts de taille}

À l'entrée de la carrière, au sud, un front de taille* (F4) orienté est-ouest se développe sur $5,5 \mathrm{~m}$ de longueur et sur plus de $1 \mathrm{~m}$ de hauteur.

Au nord, un front de taille (F1), orienté ouest-est, présente, sur presque $6 \mathrm{~m}$ de longueur et sur $1,8 \mathrm{~m}$ de hauteur, plusieurs décrochements successifs.

À l'est, le front de taille (F2), orienté nord-sud, atteint près de $9 \mathrm{~m}$ de longueur et $1,5 \mathrm{~m}$ de hauteur. Un bloc en cours d'extraction est encore en place.

Entre le front $\mathrm{F} 4$ et le front F2, un petit front secondaire (F3) est orienté nord-sud.

\section{Les déchets}

Au nord, ils se présentaient sous forme de tas qui recouvraient en partie le front de taille et empiétaient sur le sol de carrière. Certains étaient constitués de déchets d'extraction et d'éléments beaucoup plus fins, qui résultaient sans doute d'un débitage sommaire des blocs extraits. D'autres étaient constitués de blocs de grès et de sable homogène contenant une grande quantité de tessons de céramique brûlée, de verre et quelques os.

Au sud, les tas de déchets étaient beaucoup plus structurés. En bordure sud de la carrière tout d'abord, à proximité du front F2 et en face du front F3, les déchets d'extraction étaient contenus par des murets de soutènement. De la céramique datée des $\mathrm{II}^{\mathrm{e}}$ et $\mathrm{III}^{\mathrm{e}} \mathrm{s}$. ainsi qu'un fragment de broche* de tailleur de pierre y ont été recueillis.

En avant de ces murets, un tas était constitué de fragments de grès de très petites dimensions, portant souvent des marques d'outils (pic ${ }^{*}$ notamment), identifiés comme des déchets de taille.

Le long du bord sud de la carrière, des déchets d'extraction, retenus partiellement par un mur de soutènement, formaient un talus. La fouille de ce tas a révélé

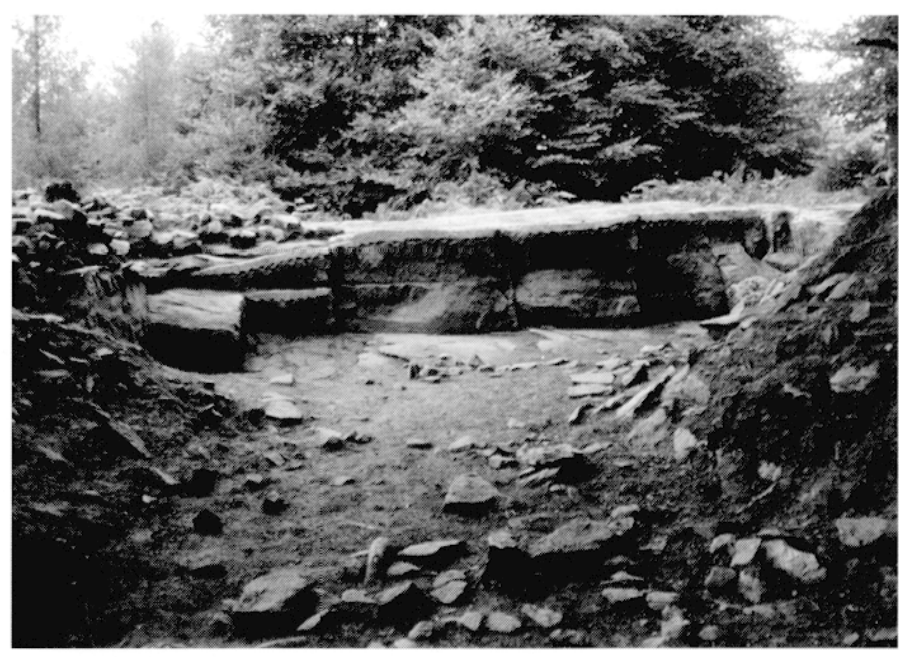

Fig. 115 - Vue générale de la carrière 1 de La Croix-Guillaume à Saint-Quirin, Moselle (photo D). Heckenbenner, musée de Sarrebourg).

la présence de fragments sculptés inachevés, dont l'exemplaire le plus significatif est un caisson funéraire en grès fissuré.

Enfin, dans la partie sud-ouest de la carrière, des déchets d'extraction étaient accumulés sans précaution, contre le front de taille F4. Une pointe de lance y a été découverte. Recouverts partiellement par ce tas, des remblais étaient constitués d'éclats de grès, de quelques tessons de céramique mélangés à du sable pulvérulent blanchâtre, et de blocs taillés dont deux sont particulièrement remarquables : une urne funéraire inachevée et une stèle-plaque incomplète portant un décor sur chaque face. D'un côté, on distingue les entrelacs d'un pampre de vigne et une grappe de raisin et, de l'autre, une corne d'abondance au-dessus d'un cartouche vraisemblablement destiné à une inscription. Le décor, particulièrement soigné, a été réalisé à la gradine*.

\section{Les aménagements à l'entrée de la carrière}

Une rampe, constituée de blocs de grès de différentes tailles et de déchets d'extraction, descend en pente douce du chemin d'accès vers le fond de la carrière. Préalablement à son installation, un essai d'extraction avait été tenté puis abandonné en raison de la mauvaise qualité du substrat. Enfin, un drain avait été aménagé pour assainir la carrière.

Entre la rampe et le front de taille F4 subsistait une cavité remplie de blocs. Son exploration a permis le dégagement d'un caniveau d'évacuation des eaux de la 
carrière, taillé dans le grès. Le canal, couvert de dalles, est dans sa première partie enterré ; il se poursuit ensuite à l'air libre, au sud, sur plusieurs mètres, pour s'interrompre sur le rebord de la terrasse.

Au-dessus du front $\mathrm{F} 4$, la roche présente deux encoches jumelées. À proximité et légèrement en contrebas, un second bloc comporte une cavité analogue quoique plus arrondie. À l'avant, scellée par des remblais, une sépulture à incinération était installée en bordure du front de taille F4, postérieurement à son exploitation.

\section{Le sol de la carrière}

Le sol de la carrière a été dégagé en totalité. Une large faille le traverse d'ouest en est. Plus profond à l'entrée de la carrière, il amorce ensuite une remontée dans sa partie nord. Les traces d'extraction sont très rares. Seules quelques marques de pics* apparaissaient çà et là. En revanche, deux coins en fer portant des marques de forge ou de leur propriétaire ont été découverts.

\section{LES TECHNIQUES D'EXTRACTION}

L'étude exhaustive des fronts de taille et des traces d'outils permet aujourd'hui d'appréhender la stratégie d'extraction adoptée sur ce site par les carriers.

La roche présente des failles et fissures verticales (lithoclases) et horizontales (joints de stratification) relativement régulières qui guident le travail du carrier. C'est une des caractéristiques des carrières de La CroixGuillaume.

Le carrier exécutait au pic $^{*}$ une encoignure* profonde de quelques centimètres ou des emboîtures ${ }^{*}$ horizontalement et verticalement afin d'introduire les coins métalliques qui lui permettaient d'isoler le bloc (fig. 116 et 117). Une fois le bloc extrait, la paroi était, si nécessaire, rectifiée au pic.

Cette technique explique l'absence quasi totale de tranchées d'extraction ${ }^{*}$ et d'emboîtures exécutées perpendiculairement à la base de la roche. De ce fait, aucune trace ne subsiste au sol, d'où la difficulté à évaluer les dimensions des blocs extraits. Toutefois, l'observation des fronts indique la hauteur et la longueur ou la largeur de ceux qui ont été exploités avant l'abandon des fronts. Ainsi le long du front F4, les blocs étaient-ils longs (de $2 \mathrm{~m}$ à $3,60 \mathrm{~m}$ ) et peu épais (de

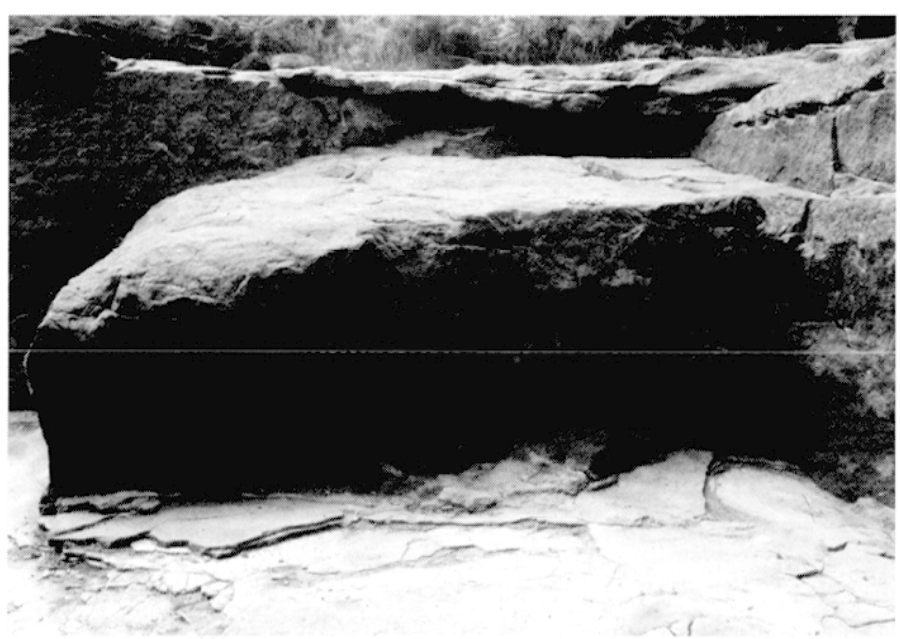

Fig. 116 - Carrière 1 de La Croix-Guillaume à Saint-Quirin, Moselle: bloc en cours d'extraction (photo D. Heckenbenner, musée de Sarrebourg).

$0,115 \mathrm{~m}$ à $0,46 \mathrm{~m}$ ), alors que les modules des éléments exploités à la fin de la période d'occupation du site étaient légèrement moins longs $(1,30 \mathrm{~m}$ à $2,40 \mathrm{~m})$ mais plus épais $(0,50 \mathrm{~m}$ à $1 \mathrm{~m})$.

Les déchets d'extraction ont été essentiellement stockés au sud du site, là où les fronts de taille n'étaient pas ou plus exploitables. Des murets de soutènement, soigneusement édifiés, les empêchaient de retomber sur le sol de la carrière. Les tas placés au nord, en revanche, ne résultent pas d'une activité d'extraction. En effet, le mobilier découvert provient soit de sépultures démantelées, soit, plus vraisemblablement, du défournement du bûcher funéraire ; ces tas ont été déposés au-dessus des derniers fronts de taille exploités, par conséquent à un moment où cette partie de la carrière ne fonctionnait plus. Il convient donc d'exclure l'hypothèse de tombes détruites par l'extraction.

L'extraction a débuté par l'ouest. Les blocs extraits ont sans doute été évacués dans un premier temps à l'aide d'un engin de levage. Les encoches creusées dans de gros blocs de grès, découverts au-dessus du front de taille F4, en seraient les vestiges partiels; il existait en effet certainement d'autres points d'ancrage qui ont aujourd'hui disparu. Ce type de machine est connu bien qu'il soit rare d'en trouver les traces (Adam, 1984, p. 46-49 ; Bessac, 1996, p. 292). Lorsque l'extraction en profondeur a été abandonnée dans la partie sud-ouest de la carrière, l'excavation a été comblée par des déchets d'extraction et une rampe a alors été construite afin d'évacuer les blocs extraits. Les blocs en attente étaient 


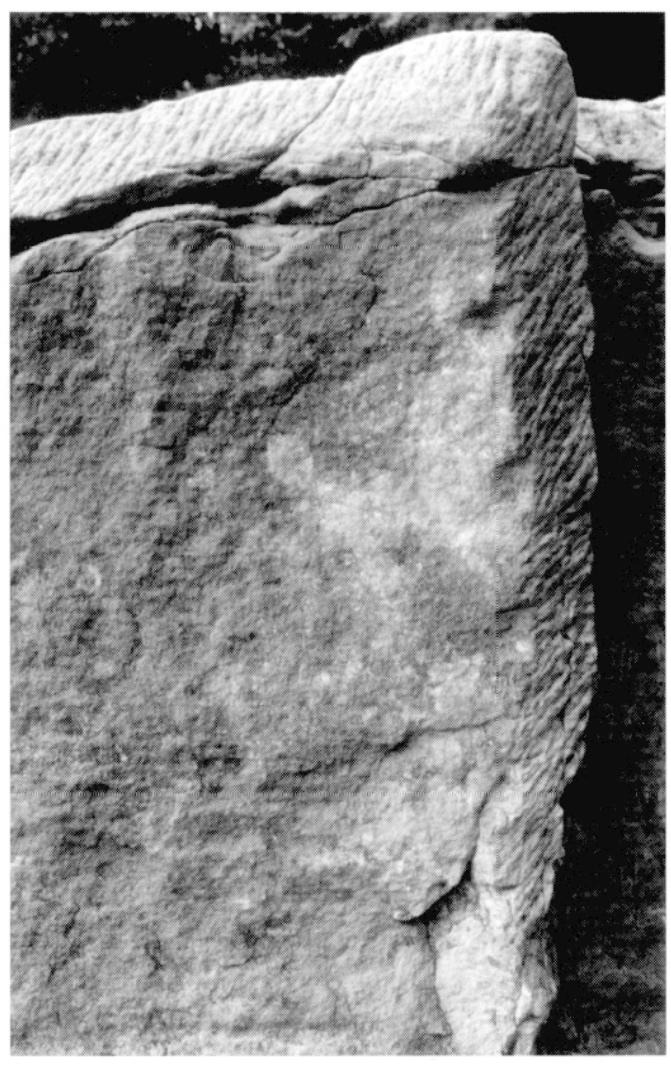

Fig. 117 - Carrière 1 de La Croix-Guillaume à Saint-Quirin, Moselle : détail d'une encoignure (photo D. Heckenbenner, musée de Sarrebourg).

certainement stockés à un endroit précis qu'il n'a pas été possible de localiser.

Dès le début de l'exploitation, les carriers ont été confrontés au problème de l'eau. Encore aujourd'hui la partie sud de la carrière est inondée durant une bonne partie de l'année. Le caniveau à l'entrée de la carrière a certainement fonctionné pendant toute la durée d'activité d'extraction. Quant au second drain, situé sous la rampe, à une cote plus élevée, il servait probablement à évacuer le trop plein d'eau.

Des calculs de volume de roche extraite ont été tentés. Il s'agit bien entendu de chiffres approximatifs puisqu'ils prennent en compte le volume de l'excavation et des déchets. On arrive ainsi à un volume total d'environ 350 à $400 \mathrm{~m}^{3}$ pour l'ensemble de l'excavation. Compte tenu de la part prise par les déchets $\left(200 \mathrm{~m}^{3}\right)$, il convient de minimiser ce résultat. On posera donc comme hypothèse que $250 \mathrm{~m}^{3} \pm 50 \mathrm{~m}^{3}$ de grès ont été extraits. Si l'on accepte un volume moyen de $2 \mathrm{~m}^{3}$ par bloc, on aurait exploité environ 100 à 150 blocs dans cette carrière.

\section{UTILISATION DES BLOCS EXTRATTS}

Les modules des blocs extraits correspondent bien aux monuments funéraires et votifs découverts dans les hameaux du secteur. Lors des fouilles précédentes, l'hypothèse d'un atelier de taille avait été soulevée à la suite de la mise au jour, à proximité de la carrière, d'une stèle funéraire, d'un fragment de stèle représentant Mercure, de deux fragments de pommes de pin et de chaperons d'enclos inachevés (B. Babault, rapport de fouille 1962-1963). Cette hypothèse est désormais confirmée grâce à la présence, au sud de la carrière, dans les tas de déchets et dans le remblai au-dessus du front de taille F4, de fragments de blocs taillés en cours d'exécution et d'une tête de broche.

Dans la nécropole également, de nombreux fragments de sculptures, dont certains inachevés, étaient réemployés, tant dans l'empierrement que dans les sépultures où ils étaient utilisés comme calage.

Cependant, les recherches concernant l'emplacement d'un atelier de taille et de sculpture n'ont pas abouti. Le nombre de ratés de taille au sud de la carrière indiquerait une localisation à proximité du front F4.

En revanche, le grès utilisé pour tailler les monuments funéraires et votifs de la nécropole et de l'aire cultuelle provient bien des carrières du site. On peut donc affirmer qu'une partie de la production était destinée à la consommation locale. Il est possible d'en dresser un inventaire sommaire : stèles en ronde bosse (Jupiter à l'anguipède, taureau, Epona), stèles votives (Mercure, divinité féminine), stèles funéraires figurées représentant des défunts en pied ou en buste, stèlesmaisons et leur soubassement, décorées ou non, de grandes ou petites dimensions $(0,50 \mathrm{~m}$ à $1,20 \mathrm{~m})$, stèlesplaques rectangulaires et stèles en battière, chaperons d'enclos, coffres funéraires ronds ou rectangulaires. La facture de la sculpture n'est pas toujours parfaite, loin s'en faut, et l'on observe des différences importantes de qualité de travail d'une stèle à l'autre.

Faut-il imputer ce fait à l'habileté plus ou moins grande du tailleur? Sans doute existait-il une hiérarchie dans ce métier; sans doute aussi, le carrier devenait-il tailleur lorsqu'il le fallait pour son propre usage ; enfin, il est plausible que l'on ait réservé aux besoins de la petite communauté de La Croix-Guillaume la production la plus modeste, et à l'extérieur les produits les plus élaborés. 
L'un des apports les plus intéressants de l'étude des fragments sculptés découverts sur le site est précisément d'avoir mis en lumière l'existence d'une production destinée à l'extérieur. En effet, quelques éléments inachevés appartiennent à des monuments funéraires, mausolées et cippes, dont on ne trouve aucune trace sur le site. La sculpture étant particulièrement soignée (utilisation d'outils variés: la broche, employée habituellement, mais aussi le ciseau et la gradine), peut-être fautil admettre l'intervention de sculpteurs itinérants à qui l'on faisait appel, lorsque le commanditaire l'exigeait.

\section{Datation}

Plusieurs arguments viennent confirmer l'antiquité de cette exploitation.

- L'implantation d'une sépulture à incinération galloromaine, au-dessus du front de taille $\mathrm{F} 4$, est postérieure à l'extraction dans ce secteur. Les cendres du défunt ont été déposées dans une urne en grès, calée par les tessons de deux cruches en céramique commune non brûlée datant de la fin du $\mathrm{I}^{\mathrm{cr}} \mathrm{s}$. Cette donnée chronologique doit être interprétée avec prudence ; d'une part, les cruches ne font pas partie du dépôt funéraire, d'autre part, l'étude de la nécropole a montré en effet que les urnes en grès étaient utilisées à partir de la seconde moitié du $\mathrm{II}^{\mathrm{e}} \mathrm{s}$. L'analyse anthropologique n'a pas permis d'expliquer la présence de cette sépulture dans la carrière.

- Les modules des blocs extraits correspondent aux modules des stèles gallo-romaines.

- Des blocs en cours de taille, datés de l'époque romaine, ont été découverts dans les déchets d'extraction, attestant une activité à cette période.

La terre de découverte, stockée dans les tas de déchets d'extraction au sud-est, contenait de la céramique du $\mathrm{II}^{\mathrm{e}} \mathrm{s}$. et du début du $\mathrm{III}^{\mathrm{e}} \mathrm{s}$. : céramique sigillée et céramique commune rugueuse produites à Mittelbronn. Cette donnée chronologique est importante puisqu'elle indique, d'une part, que l'extraction a touché un secteur occupé aux II $^{\mathrm{e}}$ et III ${ }^{\mathrm{e}} \mathrm{s}$. (il ne s'agissait pas de sépultures puisque la céramique n'était pas brûlée) et, d'autre part, que l'exploitation de cette partie de la carrière intervient à la fin de la période d'occupation du site. Les données chronologiques fournies par les autres tas de déchets doivent être interprétées avec prudence, la céramique étant mêlée aux déchets d'extraction.
L'extraction a probablement débuté au $\mathrm{II}^{\mathrm{C}} \mathrm{s}$. au plus tard. Elle s'est poursuivie jusqu'au III ${ }^{\mathbf{e}} \mathbf{s}$., peut-être parfois par intermittence, en fonction des besoins, et sans doute avec un nombre d'artisans réduit. Cette exploitation est donc fondamentalement différente des grandes carrières de la région de Nîmes dont l'activité s'est traduite de manière intensive à un moment donné et pour un chantier donné (Bessac, 1996). De ce fait, l'organisation du travail mais surtout le statut des artisans ne sont pas comparables.

\section{LES CARRIÈRES DE LA NÉCROPOLE}

\section{LA CARRIÈRE 3}

La carrière 3 appartient en quelque sorte au complexe de la carrière 1 puisqu'il s'agit du même affleurement. Elle constitue la limite sud-est de la nécropole. Il s'agit d'une modeste exploitation où quelques blocs seulement ont été extraits. Les techniques sont semblables à celles pratiquées dans la carrière 1 . Il faut pourtant souligner l'utilisation exclusive d'emboîtures*, autant que l'on puisse en juger à partir des traces subsistant sur la roche. Les modules des blocs extraits correspondraient à la fabrication de stèles-maisons.

Une sépulture avait été installée dans le comblement de la carrière. Sa datation (II ${ }^{\mathrm{e}}$ s.-début III" s.) permet ainsi de confirmer l'antiquité de l'extraction dans ce secteur.

\section{LA CARRIÈRE 7}

La petite carrière 7, dont le front présente quelques emboîtures, a été de toute évidence exploitée puis remblayée à l'époque gallo-romaine. Son comblement est en effet scellé par la couche d'abandon qui recouvrait l'ensemble de ce secteur. L'activité y a été très limitée, de l'ordre de un ou deux blocs extraits.

\section{LA CARRIÈRE 9}

Dans la partie nord du secteur central de la nécropole s'étendaient des déchets d'extraction, d'assez petite taille, utilisés, semble-t-il, pour aménager une aire de circulation. Une zone de déchets d'extraction beaucoup plus volumineux, portant parfois des traces d'outils, 
occupait toute la partie centrale du secteur. L'enlèvement de ces blocs a révélé la présence d'une carrière, vaste mais peu profonde. Une couche de sable rose-violet recouvrait le sol de la carrière. Un coin en fer et trois tessons d'une cruche ont été découverts près du front sud-est, scellés par les déchets d'extraction.

L'observation de la carrière montre que l'exploitation a commencé par le nord-ouest. Le comblement de la carrière est intervenu, semble-t-il, peu après son exploitation. L'étude des fronts révèle que les blocs exploités étaient de dimensions modestes. Les traces relevées sont peu nombreuses. La plupart sont des emboîtures, mais on observe également, au nord-ouest de la carrière, la partie inférieure d'une tranchée d'extraction.

La datation de la carrière 9 n'est pas aisée à établir. La chronologie relative apporte cependant quelques arguments en faveur d'une datation antique.

La partie nord-ouest de la carrière, c'est-à-dire la plus ancienne, a coupé un empierrement de circulation d'un secteur de la nécropole occupé au $\mathrm{I}^{\mathrm{ur}} \mathrm{s}$. et au début du II $^{\mathrm{e}} \mathrm{s}$. En revanche, en ce qui concerne la partie sud de cette carrière, aucune perturbation n'affecte le terrain de part et d'autre de l'excavation. Tout se passe comme si l'empierrement à l'est et le noyau de sépultures à l'ouest avaient évolué indépendamment l'un de l'autre. En outre, aucune sépulture n'a été touchée par l'exploitation. Les zones d'affleurement de grès sont de toute façon peu propices à l'installation de tombes.

Le début de l'exploitation de cette carrière pourrait intervenir dans la première moitié du II $^{*} \mathrm{~s}$. Il n'est pas impossible que son comblement se soit effectué au fur et à mesure de l'avancement de l'exploitation. La période de son abandon définitif est difficile à établir, mais elle ne doit pas être postérieure au début du III's. En effet, le nivellement des déchets montre que le secteur était probablement utilisé au moins à des fins de circulation. Les quelques tessons de céramique découverts à proximité du dernier front exploité, datés du II ${ }^{e}$ s., ne contredisent pas cette hypothèse.

La présence de carrières dans la nécropole n'est pas inconcevable. En effet, les zones d'affleurement ne sont pas favorables à l'implantation de sépultures. De plus, ces petites exploitations fournissaient sans doute les matériaux nécessaires à la construction des monuments funéraires, notamment des cercles de pierres, fréquents sur ce site.

\section{LES CARRIÈRES DU SECTEUR NORD}

\section{LA CARRIÈRE 4}

Située au nord du site, au-delà de la nécropole, la carrière 4 présente des caractères différents de la carrière 1.

Sa fouille partielle montre que l'exploitation du grès s'est faite en gradins du nord au sud. Les techniques d'extraction étaient toutefois semblables à celles de la carrière 1. Deux blocs, l'un en cours d'extraction, l'autre déjà extrait mais non évacué, le confirment. Après l'enlèvement des blocs, le sol était comblé de blocs de grès, permettant d'accéder aux gradins supérieurs.

Des déblais de la carrière avaient été stockés en bordure de nécropole. Mais la plupart des déchets avaient été jetés en contrebas dans le secteur déjà exploité. Un muret longeait la bordure nord du plateau. La fouille de ce secteur a montré qu'il s'agissait d'un soutènement, et non pas d'un mur de bâtiment.

En l'absence de critères de datation, il est impossible d'affirmer que cette carrière ait été exploitée dès l'Antiquité. Toutefois, la taille des blocs extraits, conforme à celle des blocs de la carrière 1 , et l'implantation de l'excavation qui a respecté le territoire de la nécropole permettent d'envisager cette hypothèse.

Une réutilisation d'une partie de cette carrière, ou du moins de ses matériaux, à la fin du XIX" ${ }^{\text {c }}$. ou au début du $\mathrm{XX}^{\mathrm{e}}$ s. est néanmoins attestée. Du mobilier de cette époque (pipes, céramiques) a en effet été découvert à proximité du muret de soutènement et à l'emplacement de traces de chariot mises en évidence entre la carrière 4 et la carrière 1. Certains blocs portent d'ailleurs des traces de taille différentes de celles qui ont été enregistrées pour l'époque romaine. Ces données sont corroborées par un texte mentionnant l'octroi à des Italiens de la parcelle forestière concernée, en vue de réempierrer un chemin (Reusch, 1911, p. 422).

\section{LA CARRIÈRE 5}

La fouille du secteur d'habitat a permis de mettre au jour le front de taille d'une vaste carrière située au nordouest du plateau. Celle-ci présente certaines caractéristiques comparables à celles de la carrière 4 , notamment le remblaiement du sol de carrière avec des blocs de grès. Parmi les éléments singuliers relevés sur les fronts de 
taille $^{*}$ figure l'utilisation d'emboîtures* de petite taille, creusées à intervalles très rapprochés. Aucun critère de datation ne permet d'affirmer que cette carrière ait fonctionné dans l'Antiquité. Toutefois, l'argumentation avancée pour la carrière 4 vaut également pour cette exploitation.

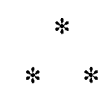

Parmi les six carrières étudiées, quatre sont datées sans ambiguité de la période romaine. En ce qui concerne les deux autres, leur cohérence topographique par rapport aux autres secteurs du site (habitat, sanctuaire, nécropole) incite à les considérer comme galloromaines, même si l'une d'entre elles au moins (carrière 4) a été réutilisée au XIX ${ }^{\mathrm{e}} \mathrm{s}$.

I'exploitation du grès a été pratiquée durant toute la durée d'occupation du site, mais il n'est pas certain qu'elle soit à l'origine de l'installation d'une population dans ce secteur. L'organisation topographique du hameau révèle toutefois l'intégration assez exceptionnelle de cette activité aux autres fonctions: habitat, nécropole, sanctuaire.

La multiplication des points d'extraction pose cependant le problème de leur contemporanéité ou de leur succession dans le temps. Dans la première hypothèse, des lots différents auraient pu être attribués aux carriers du hameau voire de hameaux voisins. Dans la seconde, les carriers auraient travaillé dans la même carrière jusqu'à épuisement complet de l'affleurement pour ensuite investir un autre lieu d'extraction. Les carrières 4 et 5 ainsi que les petites exploitations de la nécropole paraissent être en fin d'exploitation. En revanche, la carrière 1 est, semble-t-il, abandonnée alors que quelques blocs auraient pu encore être extraits. Cet abandon pourrait correspondre à la fin de l'occupation du site.

Quoi qu'il en soit, les carrières de La Croix-Guillaume correspondent à de petites unités de production qui alimentaient en grande partie le marché local. Les carriers pratiquaient probablement des activités de taille pour les besoins de la nécropole et du sanctuaire. Pour la sculpture plus élaborée, il est possible qu'ils aient fait appel à des sculpteurs itinérants. Sans doute vivaient-ils avec leur famille toute l'année dans les habitations du hameau ou à proximité : trois sites sont connus dans un rayon de 2 à $3 \mathrm{~km}$. La fouille de la totalité de la nécropole montre que trois ou quatre familles seulement vivaient sur le plateau, ce qui corroborerait l'évaluation du nombre de carriers susceptibles de travailler dans la carrière 1 par exemple, et le volume modeste qui a été extrait. Toutefois, la présence de terrasses de culture et le mobilier attestent l'existence d'activités complémentaires telles que l'agriculture, l'élevage ou la chasse.

Bien qu'aucun autre site d'extraction gallo-romain n'ait été fouillé dans ce secteur des Vosges, on peut supposer que les exploitations repérées en prospection, à l'exclusion cependant des carrières de la VIII' légion mentionnées ci-dessus, fonctionnaient de la même manière que celles de La Croix-Guillaume. 